\title{
Hypofibrinogenemia caused by tigecycline use in a patient with acute cholecystitis: a case report and review of the literature
}

\author{
Müge Ayhan ${ }^{1} \oplus$, Ferda Can $^{2} \oplus$, Selma Karaahmetoğlu ${ }^{3} \oplus$, Rahmet Güner $^{4} \odot$ \\ ${ }^{1}$ Department of Infectious Diseases and Clinical Microbiology, Ankara City Hospital, Ankara, Turkey \\ ${ }^{2}$ Department of Hematology, Ankara City Hospital, Ankara, Turkey \\ ${ }^{3}$ Department of Internal Medicine, Ankara City Hospital, Ankara, Turkey \\ ${ }^{4}$ Department of Infectious Diseases and Clinical Microbiology, Ankara Yıldırım Beyazıt University, Ankara, Turkey
}

\begin{abstract}
Objectives: Tigecycline is the first member of glicylcycline class of antibiotics, which has a broad spectrum of action. In previous reports, coagulopathy and hypofibrinogenemia caused by tigecycline use was described. We aimed to present a case of hypofibrinogenemia in association with tigecycline use. A 79-years-old male was admitted to medical intensive care unit for acute cholecystitis and acute renal failure. He had no history of coagulation disorder. He was receiving meropenem for septic shock on the admission. On the 7th day of meropenem, his infection didn't improve and fever continued. Because of that tigecycline was added to treatment. Patient's infection parameters improved, his fever dropped under treatment, but his prothrombin time, international normalized ratio and activated partial thromboplastin time levels increased and fibrinogen level decreased $(0.96 \mathrm{~g} / \mathrm{L})$. Tigecycline was discontinued that day. On the fifth day after cessasion of tigecycline, his fibrinogen levels and other coagulation parameters returned to normal ranges. The mechanisms of coagulopathy and hypofibrinogenemia should be elucidated in futher studies. We strictly suggest, regular monitoring of coagulation parameters in patients receiving tigecycline treatment.
\end{abstract}

Keywords: Tigecycline, hypofibrinogenemia, adverse effect, coagulopathy, antibiotics

$\mathrm{T}$ igecycline is the first member of glicylcycline class of antibiotics, which is structurally similar to tetracyclines. It has broad spectrum activity, particularly against multi-drug resistant bacteria (e.g Methicillin resistant Staphylococcus aureus, vancomycin resistant enterococcus, Acinetobacter baumannii) [1, 2]. It is indicated in patients who are 18 years or older for complicated intraabdominal infections, complicated skin and skin structure infections and community acquired pneumonia [3]. Tigecycline was well tolerated in registry trials, with the exception of increased rates of nausea and vomiting. But after post- marketing data signaling increased mortality rates in tigecycline treated patients have brought its use in patients with complicated infections into question, prompting other clinicians to consider other potential adverse effects which was not found in initial studies [4]. Some previous case reports showed that tigecycline seems to cause coagulation disorders, which manifested with bleeding or abnormalities in coagulation paremeters [1-5]. In this report we presented a case of hipofibrinogenemia in a patient treated with tigecycline. 


\section{CASE PRESENTATION}

A 79-year-old male who had a history of diabetes, hypertension, congestive heart failure and chronic obstructive pulmonary disease was admitted to medical intensive care unit with diagnosis of acute cholecystitis and acute renal failure from another healthcare facility. He had no history of coagulation disorder, and family history did not shown any bleeding condition. He had no underlying disorder or family history of hereditary coagulation disorder. He had been initiated on meropenem therapy for acute cholesistitis. He was on first day of meropenem and in septic shock on the admission day, and was taking noradrenaline. Blood and urine cultures were provided. Selected laboratory findings (and institutional normal ranges) were as follows: Serum creatinine level, $5.9 \mathrm{mg} / \mathrm{dL}$ (normal range 0.7-1.3 mg/dL); blood urea nitrogen $217 \mathrm{mg} / \mathrm{dL}$ (normal range 19-49 $\mathrm{mg} / \mathrm{dL}$ ); alanine aminotransferase (ALT), $340 \mathrm{U} / \mathrm{L}$ (normal range $<50 \mathrm{U} / \mathrm{L}$ ); aspartate aminotransferase (AST), $309 \mathrm{U} / \mathrm{L}$ (normal range $<50$ $\mathrm{U} / \mathrm{L}$ ); white blood cell count, 29.020 cells $/ \mathrm{mm}^{3}$ (normal range 3600-10500 cells $/ \mathrm{mm} 3$ ); hemoglobin concentration, $15 \mathrm{~g} / \mathrm{dL}$ (normal range 12.5-17.2 g/dL); platelet count $108 \times 10^{9} / \mathrm{L}$ (normal range $160-400 \times 10^{9}$ /L); INR, 1.37 (normal range 0.8-1.2). Liver enzymes of patient, C-reactive protein (CRP) $0.155 \mathrm{~g} / \mathrm{L}(0-$ $0.005 \mathrm{~g} / \mathrm{L})$ and fibrinogen level $8.7 \mathrm{~g} / \mathrm{L}(1.7-4.2 \mathrm{~g} / \mathrm{L})$ were high, INR was slightly elevated on the admission day. Peripheral blood smear on the admission showed leukocytosis with neutrophilia and platelet count was consistent with counter. On the $7^{\text {th }}$ day of meropenem, patient's acute phase reactant levels increased, his fever continued and tigecycline was added to the treatment (tigecycline dose: 100mg q24 h loading dose, $50 \mathrm{mg}$ q12 h maintenance dose) The coagulation parameters were within the normal range before tigecycline treatment. On the $14^{\text {th }}$ day of tigecycline, patient's infection improved with a dropped temperature $\left(36.2^{\circ} \mathrm{C}\right)$, white blood cell count $(9.200$ cells $/ \mathrm{mm} 3)$, platelet count $130 \times 10^{9} / \mathrm{L}$, and CRP $(0.0259 \mathrm{~g} / \mathrm{L})$. Erythrocyte morphology was normal in the peripheral smear and platelet count was consistent with $150 \times 10^{9} / \mathrm{L}$.

Nevertheless, prolonged prothrombin time (PT), INR, and activated partial thromboplastin time (aPTT) were observed; furthermore, fibrinogen levels were obviously decreased (Table 1). Liver failure findings were not observed, and the abdominal ultrasound was normal. As the patient's clinical signs of infection recovery, peripheral smear or other laboratory tests did not support disseminated intravascular coagulation, at that point we did not consider low fibrinogen, aPTT and PT elongation associated with disseminated intravascular coagulation. On that day tigecycline treatment was discontinued, meropenem was continued. After cessation of tigecycline, on the fifth day fibrinogen level became within the normal ranges and other

Table1. Laboratory parameters of patient according to time course of antimicrobial therapy

\begin{tabular}{|c|c|c|c|c|c|c|c|c|}
\hline & $\begin{array}{c}\text { Fibrinog } \\
\text { en }(g / L)\end{array}$ & aPTT (s) & $\begin{array}{l}\text { PT } \\
\text { (s) }\end{array}$ & INR & $\begin{array}{c}\text { AST } \\
(\mathbf{U} / \mathbf{L})\end{array}$ & $\begin{array}{l}\text { ALT } \\
(\mathbf{U} / \mathbf{L})\end{array}$ & $\begin{array}{l}\text { Total bilirubin } \\
\text { (mg/dL) }\end{array}$ & $\begin{array}{l}\text { CRP } \\
(\mathrm{g} / \mathrm{L})\end{array}$ \\
\hline ICU admission & 8.7 & 32.6 & 16.7 & 1.4 & 184 & 143 & 2.2 & 0.155 \\
\hline $\begin{array}{l}\text { Tigecycline } \\
\text { started }\end{array}$ & 4.16 & 23.3 & 14.6 & 1.2 & 55 & 9 & 1.8 & 0.0564 \\
\hline $\begin{array}{l}\text { 5th day of } \\
\text { tigecycline }\end{array}$ & 2.04 & 30.7 & 16.3 & 1.4 & 19 & 7 & 1.9 & 0.0815 \\
\hline $\begin{array}{l}\text { 14th day of } \\
\text { tigecycline }\end{array}$ & 0.96 & 38.9 & 18.5 & 1.6 & 28 & 16 & 1.9 & 0.0259 \\
\hline $\begin{array}{l}\text { 5th day after } \\
\text { tigecycline } \\
\text { cessation }\end{array}$ & 2.91 & 24.2 & 14.7 & 1.2 & 37 & 28 & 1.7 & 0.0731 \\
\hline $\begin{array}{l}\text { 10th day after } \\
\text { tigecycline } \\
\text { cessation }\end{array}$ & 3.18 & 24.6 & 12.9 & 1.1 & 52 & 79 & 0.9 & 0.060 \\
\hline
\end{tabular}

aPTT $=$ activated partial thromboplastin time, $\mathrm{PT}=$ prothrombin time, $\mathrm{INR}=$ international normalized ratio, $\mathrm{AST}=$ aspartate aminotransferase, $\mathrm{ALT}=$ alanin aminotransferase, $\mathrm{CRP}=\mathrm{C}$-reactive protein, $\mathrm{ICU}=$ intensive care unit 


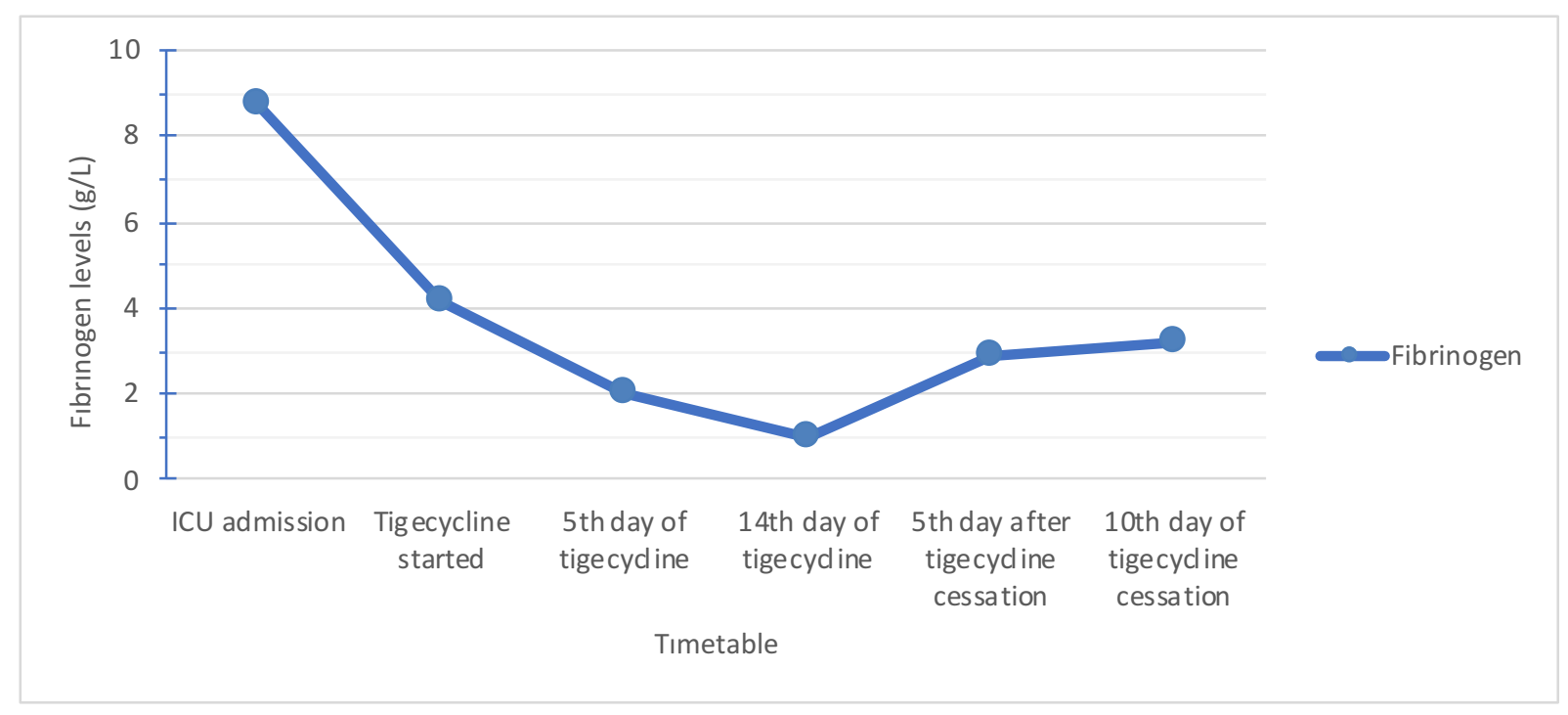

Fig. 1. Fibrinogen levels of patient according to time course of antimicrobial therapy

coagulation parameters became normal (Fig. 1). On the $67^{\text {th }}$ day of ICU admission, patient was transferred to ward and after than discharged from ward.

\section{DISCUSSION}

Tigecycline is a broad spectrum antibiotic, which is generally used for infections due to multidrug-resistant (MDR) bacteria [2]. It requires intravenous administration with a loading dose of $100 \mathrm{mg}$ followed by a maintenance dose $50 \mathrm{mg}$ every 12 hours. No dose adjustment is needed in patients with renal impairment, but only in patients with severe hepatic disfunction (Child-Pugh class $\mathrm{C}$ ), the dosage should be reduced to $25 \mathrm{mg}$ every 12 hours [1,3]. Adverse reactions, in terms of haematologic and lymphatic system, as increased partial tromboplastin time, increased PT, increased INR, eosinophilia, and trombocytopenia, might be observed during usage was stated in the instructions of tigecycline [4]. But hypofibrinogenemia was not referred and a new adverse reaction. Life threatening coagulopathy and hypofibrinogenemia cases, induced by tigecycline use, were reported in the literature, by $\mathrm{Wu}$ and $\mathrm{Wu}[1], \mathrm{Wu}$ et al. [3], Routsi et al. [6], Sabanis et al. [7], Pieringer et al. [8], Rossito et al. [9], and Y1lmaz Duran et al. [10] (Table 2).

A few clinical studies reported hypofibrinogenemia and other coagulation abnormalities caused by tigecycline use $[5,6]$. Our patient received routine dose, but in the literature some cases, which developed hypofibrinogenemia had received off-label higher doses of tigecycline $[3,6]$. The mechanism in which tigecycline induced coagulopathy and caused hypofibrinogenemia, is unknown. Fibrinogen is produced by hepatocytes. It could be converted to insoluble fibrin to form blood clots, when trauma or sepsis occurs [1]. Effects of vitamin $\mathrm{K}$ deficiency on gut flora and inflammation due to serious infections are also commonly cited mechanisms resulting in coagulopathy. However, vitamin K replacement is reported not to improve coagulopathy, which is caused by tigecycline use [2]. In our case, serious infection might be thought to cause hypofibrinogenemia, but patient's infection parameters improved when fibrinogen level started to decrease. Furthermore, effect of tigecycline on liver functions could implicate decreased levels of fibrinogen [7]. Therefore, the underlying mechanisms of coagulopathy and hypofibrinogenemia and risk factors for these adverse effects should be elucidated. Also we suggest, regular monitoring of coagulation parameters, including fibrinogen level in patients receiving tigecycline. If patients develop hypofibrinogenemia, discontinuation of drug should be considered.

\section{CONCLUSION}

We presented a patient who developed hypofibrinogenemia because of tigecycline use. The underlying mechanisms of coagulopathy and hypofibrinogenemia and risk factors for these adverse 


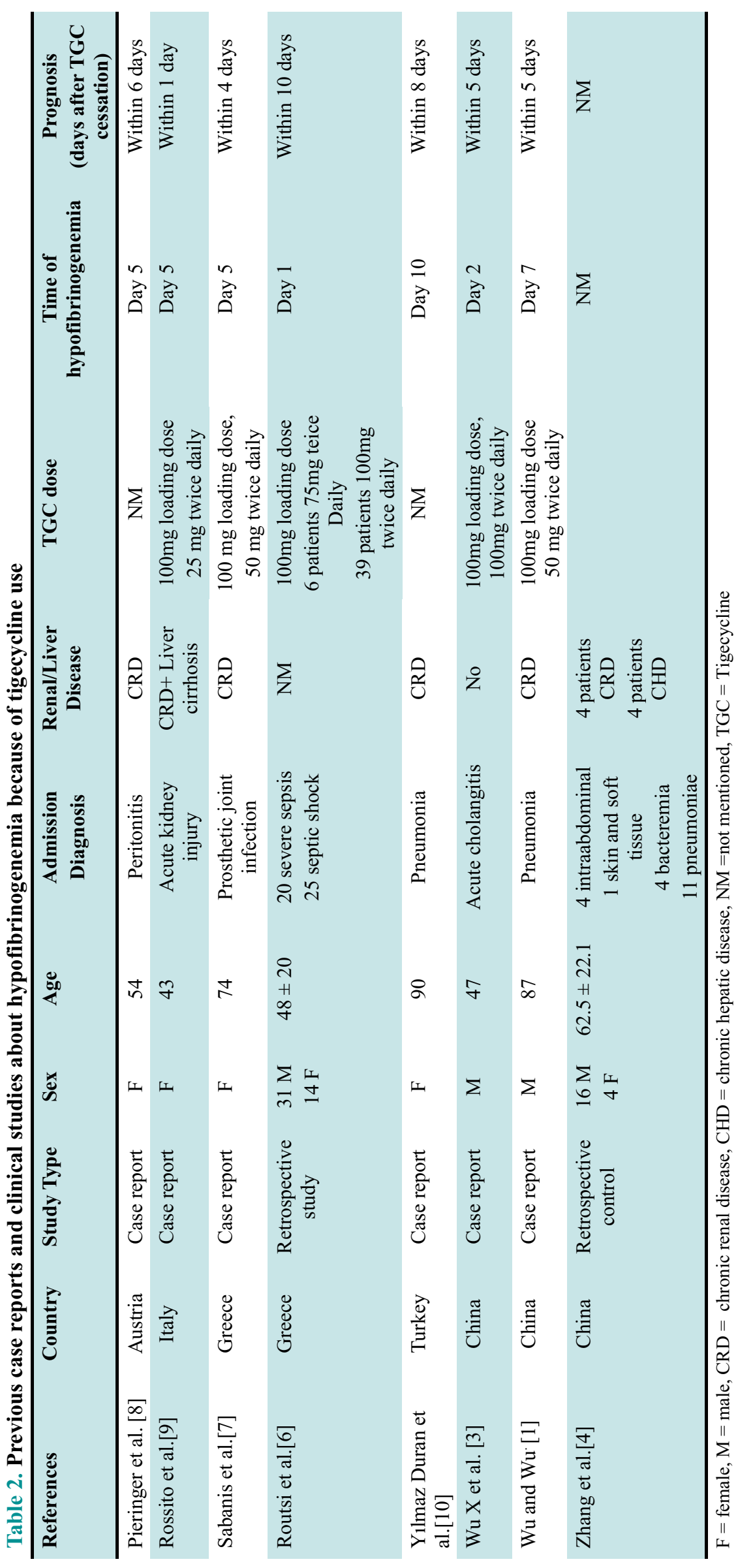


effects should be elucidated. We suggest, regular monitoring of coagulation parameters including fibrinogen level in patients receiving tigecycline. If patients develop hypofibrinogenemia, discontinuation of drug should be considered.

\section{Authors' Contribution}

Study Conception: HRG, MA, SK, FC; Study Design: RG, MA, SK, FC; Supervision: RG, MA, SK, FC; Fundings: MA; Materials: MA, SK, FC; Data Collection and/or Processing: MA; Statistical Analysis and/or Data Interpretation: MA, RG; Literature Review: MA; Manuscript Preparation: MA and Critical Review: MA, RG.

\section{Informed consent}

Written informed consent was obtained from the patient for publication of this case and any accompanying images.

\section{Conflict of interest}

The authors disclosed no conflict of interest during the preparation or publication of this manuscript.

\section{REFERENCES}

1. Wu PC, Wu CC. Tigecycline-associated hypofibrinogenemia: a case report and review of the literature. IDCases 2018;11:56-7.

2. Cui N, Cai H, Li Z, Lu Y, Wang G, Lu A. Tigecycline-induced coagulopathy: a literature review. Int J Clin Pharm 2019;41:1408-13.
3. Wu X, Zhao P, Dong L, Zhang X. A case report of patient with severe acute cholangitis with tigecycline treatment causing coagulopathy and hypofibrinogenemia. Med (United States) 2017;96:2016-8.

4. Zhang Q, Zhou S, Zhou J. Tigecycline treatment causes a decrease in fibrinogen levels. Antimicrob Agents Chemother 2015;59:1650-5.

5. Zhang J, Yu L, Fu Y, Zhao Y, Wang Y, Zhao J, et al. Tigecycline in combination with other antibiotics against clinical isolates of carbapenem-resistant klebsiella pneumoniae in vitro. Ann Palliat Med 2019;8:522-631.

6. Routsi C, Kokkoris S, Douka E, Ekonomidou F, Karaiskos I, Giamarellou H. High-dose tigecyclineassociated alterations in coagulation parameters in critically ill patients with severe infections. Int J Antimicrob Agents 2015;45:90-3.

7. Sabanis N, Paschou E, Gavriilaki E, Kalaitzoglou A, Vasileiou S. Hypofibrinogenemia induced by tigecycline: a potentially life-threatening coagulation disorder. Infect Dis (Lond) 2015;47:743-6.

8. Pieringer H, Schmekal B, Biesenbach G, Pohanka E. Severe coagulation disorder with hypofibrinogenemia associated with the use of tigecycline. Ann Hematol 2010;89:1063-4.

9. Rossitto G, Piano S, Rosi S, Simioni P, Angeli P. Life-threatening coagulopathy and hypofibrinogenaemia induced by tigecycline in a patient with advanced liver cirrhosis. Eur J Gastroenterol Hepatol 2014;26:681-4.

10. Yılmaz Duran F, Yildırım H, Şen EM. A lesser known side effect of tigecycline: hypofibrinogenemia. Turkish J Hematol 2018;35:83-4. 